Dermatology 2020;236:179-182

DOI: $10.1159 / 000502642$

\section{Association of Serum Vitamins with Eczema in US Adults (NHANES 2005-2006)}

\author{
Jia Wei John S. Jia, b \\ ${ }^{a}$ Environmental Research Center, Duke Kunshan University,

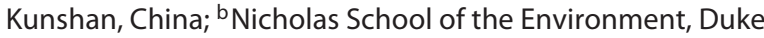 \\ University, Durham, NC, USA
}

Keywords

Eczema $\cdot$ Vitamins $\cdot$ NHANES

\section{Dear editor,}

Eczema is one of the most common dermatological issue, with dermatitis being the highest contributor of disability-adjusted life years (DALYs) to global skin disease morbidity and mortality [1]. We hypothesize that vitamins may be involved in the etiology of eczema, through their influence on the epidermis or the immune system. We tested our hypothesis cross-sectionally in the general population of the USA.

We used the US National Health and Nutrition Examination Survey (NHANES) conducted in 2005-2006. NHANES is a series of national, population-based, cross-sectional surveys conducted by the National Center for Health Statistics. The NHANES study sample was selected to represent the general non-institutionalized US population [2]. We included adults 20 years and older. Ever-report of eczema was assessed through the question "Has a doctor or other health professional ever told you that you have eczema?" Serum concentrations of vitamin D (25-hydroxyvitamin D), vitamin A (retinol), vitamin $E$ ( $\alpha$ and $\gamma$-tocopherol), vitamin $B_{6}$ (pyridoxal $5^{\prime}$-phosphate), and vitamin $\mathrm{B}_{12}$ were measured. The association between eczema and tertiles of serum vitamin levels was estimated using multivariate logistic regression models adjusted for patient demographic variables (age, sex, season, race, education, income), lifestyle variables (BMI, smoking, drinking), stress variables (depression, sleep), and medical co-morbidities (asthma, hay fever, IgE). A sample weight was assigned to each individual representative of the US Census population, which is a measure of the number of people in the population represented by that sample person. We used SAS version 9.4 for regression models to estimate ORs with $95 \%$ CI. The ORs represented the odds of report of eczema versus no report of eczema. Results were considered significant at a level of $p<0.05$.

A total of 3,925 adults were included in the analysis. The prevalence of ever-report of eczema was 7.9\% (Table 1). Comparing the lowest with the highest tertile (reference category) of vitamin concentrations, we did not see a relationship of vitamin A (OR 1.26, 95\% CI 0.86-1.83), vitamin E (OR 1.34, 95\% CI 0.81-2.20), vitamin $\mathrm{B}_{6}$ (OR 1.07, 95\% CI 0.72-1.60), or vitamin $\mathrm{B}_{12}$ (OR 1.25, 95\% CI $0.74-2.10)$ with risk of eczema. We saw a protective effect of vitamin $\mathrm{D}$, with the lowest (range 13.2-47.1 nmol/L) and middle (range 49.5-66.5 nmol/L) tertiles of vitamin D showing higher risk of eczema (OR 1.70, 95\% CI 1.05-2.74; OR 1.45, 95\% CI 0.99-2.11) when compared to the highest tertile (range 68.9-195 nmol/L; Table 2).

A previous study in Korea reported that low vitamin D level was associated with eczema in an adult population [3]. A randomized, double-blinded, placebo-controlled study of atopic dermatitis patients found that vitamin $\mathrm{D}$ supplementation resulted in significant improvements in atopic dermatitis severity [4]. However, a UK Biobank study found no association between genetically lowered vitamin D levels and atopic dermatitis [5]. The relationship between vitamin $\mathrm{D}$ and eczema is still unclear. Our study found a relationship between vitamin D (serum 25-hydroxyvitamin D) level and the reports of eczema, which appears to have a dose-response relationship. Our finding provides additional evidence that some vitamin deficiencies are possible risk factors in the development of eczema. Our study has several limitations. First, NHANES is a cross-sectional survey, which cannot infer a causal relationship. Second, self-reporting eczema is subjected to a potential of recall bias. Disease severity and healthcare utilization may also underrepresent the true prevalence of eczema. Third, because the survey question was about ever-report of eczema, we do not know the vitamin levels when the patients had active disease, which may weaken the association. Considering that there is no consensus on using vitamins for eczema prevention and treatment in the current clinical guidelines, vitamin supplementation, in relationship with serum vitamin levels, especially vitamin $\mathrm{D}$, needs to be elucidated in future studies.

\section{Key Message}

Vitamin D, but not other vitamins, might have an association with eczema in US adults.

\section{Disclosure Statement}

The authors have no conflicts of interest to declare.

Funding Sources

No funding was received for this study.

\section{Author Contributions}

J.W. and J.S.J. designed the study. J.W. had access to raw data and conducted statistical analysis. J.W. and J.S.J. interpreted the results. Both authors contributed to the paper and approved of the final version.
John S. Ji, ScD

Environmental Health Science Duke Kunshan University, 8 Duke Avenue Kunshan, Jiangsu 215316 (China)

E-Mail john.ji@dukekunshan.edu.cn 
Table 1. Eczema prevalence and serum vitamin levels according to demographic, lifestyle, stress, and medical co-morbidity

\begin{tabular}{|c|c|c|c|c|c|c|c|c|c|}
\hline Total & $\begin{array}{l}\text { Eczema, } \\
n(\%)\end{array}$ & $\begin{array}{l}\text { No eczema, } \\
n(\%)\end{array}$ & $\begin{array}{l}\text { OR } \\
(95 \% \mathrm{CI})\end{array}$ & $p$ value & $\begin{array}{l}\text { Vitamin D, } \\
\mathrm{nmol} / \mathrm{L}\end{array}$ & $\begin{array}{l}\text { Vitamin A, } \\
\mu \mathrm{mol} / \mathrm{L}\end{array}$ & $\begin{array}{l}\text { Vitamin } \mathrm{E}, \\
\mu \mathrm{mol} / \mathrm{L}\end{array}$ & $\begin{array}{l}\text { Vitamin } \mathrm{B}_{6} \\
\mathrm{nmol} / \mathrm{L}\end{array}$ & $\begin{array}{l}\text { Vitamin } \mathrm{B}_{12} \\
\mathrm{pmol} / \mathrm{L}\end{array}$ \\
\hline Overall, $n(\%)$ & $265(7.9)$ & $3,660(92.1)$ & & & $61.3(1.1)$ & $2.12(0.02)$ & $29.3(0.4)$ & $76.9(2.8)$ & $401.5(8.3)$ \\
\hline Age, years & & & & & {$[0.046]$} & {$[<0.001]$} & {$[0.07]$} & {$[0.0003]$} & {$[<0.001]$} \\
\hline Mean & $45.4(1.2)$ & $46.6(0.8)$ & & 0.36 & & & & & \\
\hline $\operatorname{Sex}$ & & & & & {$[0.54]$} & {$[<0.001]$} & {$[0.07]$} & {$[0.03]$} & {$[0.001]$} \\
\hline Female & $154(9.3)$ & $1,877(90.7)$ & 1 (ref.) & & $61.5(1.3)$ & $2.01(0.02)$ & $30.0(0.5)$ & $73.1(2.2)$ & $417.8(13.5)$ \\
\hline Male & $111(6.5)$ & $1,783(93.5)$ & $0.67(0.53-0.84)$ & 0.002 & $61.1(1.0)$ & $2.23(0.02)$ & $28.6(0.3)$ & $81.0(4.5)$ & $384.2(6.9)$ \\
\hline Season at sampling & & & & & {$[<0.001]$} & {$[0.90]$} & {$[0.24]$} & {$[0.18]$} & {$[0.48]$} \\
\hline Winter & $82(5.4)$ & $1,682(94.6)$ & 1 (ref.) & & $55.6(1.4)$ & $2.12(0.03)$ & $29.6(0.4)$ & $73.9(3.1)$ & $421.5(19.7)$ \\
\hline Summer & $183(9.7)$ & $1,972(90.3)$ & $1.90(1.19-3.04)$ & 0.01 & $65.2(1.0)$ & $2.12(0.02)$ & $29.2(0.4)$ & $78.9(1.5)$ & $387.6(7.5)$ \\
\hline Race/ethnicity & & & & & {$[<0.001]$} & {$[0.89]$} & {$[0.14]$} & {$[0.04]$} & {$[0.03]$} \\
\hline Mexican American & $13(1.9)$ & $757(98.1)$ & $0.20(0.13-0.30)$ & $<0.001$ & $51.9(2.0)$ & $1.88(0.02)$ & $27.7(0.5)$ & $67.2(3.0)$ & $570.8(70.7)$ \\
\hline Other Hispanic & $6(4.7)$ & $115(95.3)$ & $0.51(0.23-1.14)$ & 0.01 & $53.6(1.4)$ & $1.94(0.03)$ & $27.7(0.5)$ & $74.1(4.7)$ & $408.9(11.9)$ \\
\hline Non-Hispanic white & $176(8.9)$ & $1,862(91.1)$ & 1 (ref.) & & $65.8(1.1)$ & $2.20(0.02)$ & $30.4(0.4)$ & $81.3(3.8)$ & $382.5(7.5)$ \\
\hline Non-Hispanic black & $60(6.9)$ & $793(93.1)$ & $0.77(0.54-1.08)$ & 0.12 & $41.9(1.3)$ & $1.90(0.02)$ & $24.7(0.3)$ & $58.6(4.3)$ & $425.8(10.2)$ \\
\hline Other & $10(7.0)$ & $133(93.0)$ & $0.77(0.45-1.32)$ & 0.31 & $54.9(1.9)$ & $1.91(0.06)$ & $26.9(0.7)$ & $65.5(5.8)$ & $369.1(18.9)$ \\
\hline Education & & & & & {$[<0.001]$} & {$[<0.001]$} & {$[<0.001]$} & {$[0.16]$} & {$[<0.001]$} \\
\hline Less than 9th grade & $12(3.2)$ & $429(96.8)$ & 1 (ref.) & & $55.7(1.3)$ & $2.06(0.03)$ & $29.8(0.7)$ & $61.1(4.0)$ & $592.8(115.3)$ \\
\hline 9th-11th grade & $31(6.8)$ & $558(93.2)$ & $2.20(0.80-6.04)$ & 0.12 & $56.2(2.0)$ & $2.01(0.04)$ & $26.5(0.5)$ & $56.0(3.6)$ & $387.7(11.6)$ \\
\hline High school graduate & $59(7.8)$ & $877(92.2)$ & $2.55(1.27-5.12)$ & 0.01 & $60.3(1.2)$ & $2.13(0.03)$ & $28.9(0.7)$ & $70.8(3.6)$ & $387.9(13.1)$ \\
\hline Some college or associate degree & $90(8.3)$ & $1,043(91.7)$ & $2.76(1.19-6.40)$ & 0.02 & $62.0(1.3)$ & $2.11(0.03)$ & $29.0(0.5)$ & $75.6(3.2)$ & $383.9(9.8)$ \\
\hline College graduate and above & $73(9.1)$ & $753(90.9)$ & $3.05(1.17-7.94)$ & 0.03 & $64.7(1.1)$ & $2.18(0.03)$ & $31.1(0.6)$ & $95.7(7.0)$ & $400.0(10.0)$ \\
\hline Income (PIR) & & & & & {$[<0.001]$} & {$[<0.001]$} & {$[<0.001]$} & {$[0.007]$} & {$[<0.001]$} \\
\hline $0-1.85$ & $84(6.3)$ & $1,411(93.7)$ & 1 (ref.) & & $56.7(1.5)$ & $2.03(0.02)$ & $27.3(0.4)$ & $60.2(1.9)$ & $406.0(10.7)$ \\
\hline $1.86-3.50$ & $68(7.8)$ & $993(92.2)$ & $1.24(0.76-2.04)$ & 0.36 & $60.8(1.3)$ & $2.12(0.02)$ & $29.2(0.5)$ & $75.0(5.0)$ & $419.8(28.5)$ \\
\hline$>3.50$ & $113(9.0)$ & $1,256(91.0)$ & $1.45(1.00-2.11)$ & 0.05 & $64.3(1.3)$ & $2.17(0.02)$ & $30.6(0.4)$ & $87.6(3.8)$ & $387.8(5.9)$ \\
\hline Smoking & & & & & {$[0.36]$} & {$[0.28]$} & {$[<0.001]$} & {$[0.004]$} & {$[<0.001]$} \\
\hline Never smoker & $126(7.4)$ & $1,912(92.6)$ & 1 (ref.) & & $60.5(1.3)$ & $2.08(0.02)$ & $29.7(0.5)$ & $83.6(3.3)$ & $422.8(14.5)$ \\
\hline Past smoker & $73(9.0)$ & $951(91.0)$ & $1.23(0.85-1.80)$ & 0.26 & $62.7(1.3)$ & $2.24(0.03)$ & $31.3(0.3)$ & $83.1(4.6)$ & $405.9(10.0)$ \\
\hline Current smoker & $66(7.9)$ & $797(92.1)$ & $1.06(0.78-1.45)$ & 0.67 & $61.5(1.2)$ & $2.08(0.03)$ & $26.3(0.4)$ & $56.2(3.1)$ & $352.0(9.0)$ \\
\hline Drinking & & & & & {$[<0.001]$} & {$[<0.001]$} & {$[0.84]$} & {$[0.14]$} & {$[0.01]$} \\
\hline Never drinker & $25(5.5)$ & $506(94.5)$ & 1 (ref.) & & $57.0(2.0)$ & $1.97(0.04)$ & $29.1(0.8)$ & $67.6(3.5)$ & $419.7(14.5)$ \\
\hline Past drinker & $43(5.9)$ & $778(94.1)$ & $1.07(0.53-2.15)$ & 0.84 & $57.5(1.1)$ & $2.07(0.03)$ & $30.4(0.8)$ & $83.5(6.7)$ & $415.4(14.2)$ \\
\hline Current drinker light & $76(10.1)$ & $793(89.9)$ & $1.94(0.97-3.90)$ & 0.06 & $61.3(1.2)$ & $2.12(0.03)$ & $31.1(0.5)$ & $78.8(3.4)$ & $410.8(11.7)$ \\
\hline Current drinker heavy & $121(8.1)$ & $1,583(91.9)$ & $1.52(0.81-2.83)$ & 0.18 & $63.6(1.2)$ & $2.17(0.02)$ & $28.1(0.3)$ & $75.7(3.1)$ & $387.9(15.3)$ \\
\hline$B M I, \mathrm{~kg} / \mathrm{m}^{2}$ & & & & & {$[<0.001]$} & {$[0.71]$} & {$[<0.001]$} & {$[0.09]$} & {$[<0.001]$} \\
\hline Underweight & $3(3.9)$ & $63(96.1)$ & $0.37(0.09-1.58)$ & 0.17 & $61.9(3.6)$ & $1.80(0.04)$ & $24.3(1.4)$ & $65.4(7.7)$ & $482.6(52.0)$ \\
\hline Normal & $87(9.9)$ & $1,016(90.1)$ & 1 (ref.) & & $66.5(1.2)$ & $2.09(0.03)$ & $27.8(0.5)$ & $84.1(4.1)$ & $400.2(5.4)$ \\
\hline Overweight & $73(6.2)$ & $1,287(93.8)$ & $0.60(0.39-0.94)$ & 0.03 & $62.7(1.3)$ & $2.21(0.02)$ & $30.9(0.5)$ & $89.0(5.6)$ & $420.9(21.3)$ \\
\hline Obesity & $102(8.1)$ & $1,294(91.9)$ & $0.81(0.62-1.05)$ & 0.10 & $55.4(1.1)$ & $2.08(0.02)$ & $29.4(0.4)$ & $59.8(3.0)$ & $380.2(13.9)$ \\
\hline Sleep & & & & & {$[0.001]$} & {$[0.05]$} & {$[0.08]$} & {$[0.38]$} & {$[0.02]$} \\
\hline$<6$ hours & $41(7.4)$ & $545(92.6)$ & $0.93(0.63-1.37)$ & 0.68 & $54.7(1.5)$ & $2.07(0.03)$ & $27.7(0.6)$ & $64.2(3.4)$ & $428.4(46.9)$ \\
\hline $6-7 \mathrm{~h}$ & $130(7.9)$ & $1,823(92.1)$ & 1 (ref.) & & $61.9(1.1)$ & $2.12(0.02)$ & $29.4(0.5)$ & $76.9(2.1)$ & $398.9(7.9)$ \\
\hline $7+h$ & $94(8.2)$ & $1,292(91.8)$ & $1.04(0.63-1.72)$ & 0.88 & $63.0(1.3)$ & $2.14(0.02)$ & $29.9(0.4)$ & $81.9(6.1)$ & $395.2(7.6)$ \\
\hline Depression & & & & & {$[0.03]$} & {$[0.94]$} & {$[0.003]$} & {$[0.43]$} & {$[0.04]$} \\
\hline Never & $190(7.1)$ & $2,939(92.9)$ & 1 (ref.) & & $61.8(1.1)$ & $2.12(0.02)$ & $29.5(0.4)$ & $79.5(2.7)$ & $396.1(6.6)$ \\
\hline Mild & $38(9.0)$ & $512(91.0)$ & $1.29(0.69-2.40)$ & 0.39 & $60.3(2.0)$ & $2.08(0.03)$ & $29.6(0.6)$ & $72.0(7.3)$ & $400.1(17.3)$ \\
\hline Moderate-severe & $37(17.6)$ & $209(82.4)$ & $2.80(1.96-4.00)$ & $<0.001$ & $55.8(2.0)$ & $2.17(0.06)$ & $26.6(0.9)$ & $51.7(5.6)$ & $485.7(112.6)$ \\
\hline
\end{tabular}


Table 1. (continued)

\begin{tabular}{|c|c|c|c|c|c|c|c|c|c|}
\hline Total & $\begin{array}{l}\text { Eczema, } \\
n(\%)\end{array}$ & $\begin{array}{l}\text { No eczema, } \\
n(\%)\end{array}$ & $\begin{array}{l}\text { OR } \\
(95 \% \mathrm{CI})\end{array}$ & $p$ value & $\begin{array}{l}\text { Vitamin D, } \\
\mathrm{nmol} / \mathrm{L}\end{array}$ & $\begin{array}{l}\text { Vitamin A, } \\
\mu \mathrm{mol} / \mathrm{L}\end{array}$ & $\begin{array}{l}\text { Vitamin E, } \\
\mu \mathrm{mol} / \mathrm{L}\end{array}$ & $\begin{array}{l}\text { Vitamin } \mathrm{B}_{6} \text {, } \\
\mathrm{nmol} / \mathrm{L}\end{array}$ & $\begin{array}{l}\text { Vitamin } \mathrm{B}_{12} \text {, } \\
\mathrm{pmol} / \mathrm{L}\end{array}$ \\
\hline$I g E, k U / L$ & & & & & {$[<0.001]$} & {$[0.002]$} & {$[0.02]$} & {$[0.96]$} & {$[<0.001]$} \\
\hline Tertile 1 (1.41-24.00) & $79(7.3)$ & $1,230(92.7)$ & 1 (ref.) & & $63.6(1.2)$ & $2.15(0.02)$ & $30.5(0.4)$ & $80.4(5.1)$ & $398.8(8.2)$ \\
\hline Tertile $2(24.10-90.30)$ & $99(8.9)$ & $1,210(91.1)$ & $1.25(0.85-1.83)$ & 0.23 & $61.7(1.0)$ & $2.12(0.03)$ & $29.0(0.5)$ & $80.3(2.3)$ & $407.2(22.8)$ \\
\hline Tertile $3(90.5-16,148)$ & $87(7.6)$ & $1,220(92.4)$ & $1.05(0.77-1.44)$ & 0.73 & $57.7(1.4)$ & $2.08(0.02)$ & $28.1(0.4)$ & $68.1(3.1)$ & $398.5(11.1)$ \\
\hline Asthma & & & & & {$[0.59]$} & {$[0.03]$} & {$[<0.001]$} & {$[0.16]$} & {$[0.002]$} \\
\hline Never & $208(7.1)$ & $3,206(92.9)$ & 1 (ref.) & & $60.8(1.84)$ & $2.13(0.02)$ & $29.6(0.4)$ & $79.0(2.9)$ & $405.2(9.4)$ \\
\hline Ever & $57(12.9)$ & $454(87.1)$ & $1.93(1.31-2.84)$ & 0.003 & $61.4(1.01)$ & $2.06(0.03)$ & $27.7(0.5)$ & $64.0(4.1)$ & $379.0(14.6)$ \\
\hline Hay fever & & & & & {$[0.01]$} & {$[0.003]$} & {$[0.44]$} & {$[0.56]$} & {$[0.01]$} \\
\hline Never & $206(6.9)$ & $3,300(93.1)$ & 1 (ref.) & & $64.4(1.60)$ & $2.11(0.02)$ & $29.1(0.3)$ & $76.3(3.1)$ & $403.1(8.7)$ \\
\hline Ever & $59(14.9)$ & $360(85.1)$ & $2.39(1.54-3.69)$ & 0.001 & $60.8(1.08)$ & $2.19(0.03)$ & $30.9(0.8)$ & $80.6(4.8)$ & $391.4(18.6)$ \\
\hline
\end{tabular}

Eczema prevalence values are weighted. Serum vitamin levels are presented as mean (SEM). Values in square brackets represent $p$ values. PIR, poverty-to-income ratio.

Table 2. Predicted ORs and 95\% CIs of eczema according to serum vitamin D, A, E, $\mathrm{B}_{6}$, and $\mathrm{B}_{12}$ levels (tertiles)

\begin{tabular}{|c|c|c|c|c|c|c|c|c|c|c|c|}
\hline & $n$ & Crude & $p$ value & Model 1 & $p$ value & Model 2 & $p$ value & Model 3 & $p$ value & Model 4 & $p$ value \\
\hline \multicolumn{12}{|c|}{ Vitamin $D, \mathrm{nmol} / \mathrm{L}$} \\
\hline $13.2-47.1$ & 1,352 & $1.08(0.72-1.62)$ & 0.70 & $1.60(1.00-2.56)$ & 0.05 & $1.67(1.05-2.67)$ & 0.03 & $1.64(1.04-2.58)$ & 0.03 & $1.70(1.05-2.74)$ & 0.03 \\
\hline $49.5-66.5$ & 1,408 & $1.21(0.84-1.75)$ & 0.27 & $1.46(0.99-2.13)$ & 0.05 & $1.49(1.01-2.13)$ & 0.04 & $1.43(0.98-2.11)$ & 0.07 & $1.45(0.99-2.11)$ & 0.05 \\
\hline 68.9-195 & 1,161 & 1 (ref.) & & 1 (ref.) & & 1 (ref.) & & 1 (ref.) & & 1 (ref.) & \\
\hline \multicolumn{12}{|c|}{ Vitamin $A, \mu \mathrm{mol} / \mathrm{L}$} \\
\hline $0.024-1.746$ & 1,304 & $1.19(0.75-1.90)$ & 0.43 & $1.17(0.79-1.72)$ & 0.44 & $1.19(0.81-1.74)$ & 0.37 & $1.22(0.84-1.77)$ & 0.29 & $1.26(0.86-1.83)$ & 0.23 \\
\hline $1.749-2.238$ & 1,282 & $1.17(0.79-1.73)$ & 0.40 & $1.14(0.79-1.63)$ & 0.49 & $1.14(0.79-1.66)$ & 0.49 & $1.18(0.82-1.70)$ & 0.37 & $1.19(0.82-1.72)$ & 0.36 \\
\hline $2.241-6.458$ & 1,289 & 1 (ref.) & & 1 (ref.) & & 1 (ref.) & & 1 (ref.) & & 1 (ref.) & \\
\hline \multicolumn{12}{|c|}{ Vitamin $E, \mu m o l / L$} \\
\hline $7.245-23.011$ & 1,292 & $1.32(0.82-2.12)$ & 0.24 & $1.40(0.84-2.32)$ & 0.20 & $1.37(0.83-2.28)$ & 0.22 & $1.32(0.80-2.18)$ & 0.28 & $1.34(0.81-2.20)$ & 0.25 \\
\hline $23.034-30.883$ & 1,313 & $0.77(0.59-1.02)$ & 0.07 & $0.76(0.55-1.06)$ & 0.10 & $0.74(0.54-1.03)$ & 0.07 & $0.73(0.52-1.01)$ & 0.05 & $0.73(0.52-1.04)$ & 0.08 \\
\hline $31.115-137.23$ & 1,270 & 1 (ref.) & & 1 (ref.) & & 1 (ref.) & & 1 (ref.) & & 1 (ref.) & \\
\hline \multicolumn{12}{|c|}{ Vitamin $B_{6}, n m o l / L$} \\
\hline $2.0-31.0$ & 1,306 & $1.11(0.73-1.67)$ & 0.60 & $1.16(0.76-1.77)$ & 0.50 & $1.16(0.77-1.75)$ & 0.49 & $1.08(0.70-1.67)$ & 0.73 & $1.07(0.72-1.60)$ & 0.73 \\
\hline $31.1-64.0$ & 1,306 & $1.11(0.80-1.54)$ & 0.49 & $1.20(0.92-1.57)$ & 0.19 & $1.20(0.91-1.57)$ & 0.19 & $1.18(0.88-1.57)$ & 0.27 & $1.17(0.87-1.56)$ & 0.29 \\
\hline $64.1-1,740$ & 1,304 & 1 (ref.) & & 1 (ref.) & & 1 (ref.) & & 1 (ref.) & & 1 (ref.) & \\
\hline \multicolumn{12}{|c|}{ Vitamin $B_{12}, \mathrm{pmol} / \mathrm{L}$} \\
\hline $36.9-281.92$ & 1,283 & $1.32(0.76-2.27)$ & 0.30 & $1.28(0.76-2.17)$ & 0.34 & $1.28(0.75-2.18)$ & 0.36 & $1.27(0.76-2.12)$ & 0.36 & $1.25(0.74-2.10)$ & 0.41 \\
\hline $282.65-419.92$ & 1,274 & $0.96(0.55-1.67)$ & 0.86 & $0.94(0.55-1.58)$ & 0.81 & $0.93(0.55-1.58)$ & 0.79 & $0.93(0.56-1.56)$ & 0.79 & $0.89(0.53-1.49)$ & 0.65 \\
\hline $420.66-51,807.6$ & 1,275 & 1 (ref.) & & 1 (ref.) & & 1 (ref.) & & 1 (ref.) & & 1 (ref.) & \\
\hline
\end{tabular}

Model 1: adjusted for demographic variables (age, sex, season, race, education, income). Model 2: adjusted for demographic and lifestyle variables (BMI, smoking, drinking). Model 3: adjusted for demographic, lifestyle, and stress variables (depression, sleep). Model 4: adjusted for demographic, lifestyle, stress, and medical co-morbidity variables (asthma, hay fever, IgE). 


\section{References}

1 Karimkhani C, Dellavalle RP, Coffeng LE, Flohr C, Hay RJ, Langan SM, et al. Global Skin Disease Morbidity and Mortality: An Update From the Global Burden of Disease Study 2013. JAMA Dermatol. 2017 May; 153(5):406-12.

2 CDC. About the National Health and Nutrition Examination Survey (Introduction) 2017 [updated Sep 15, 2017]. Available from: https://www. cdc.gov/nchs/nhanes/about_nhanes.htm.

3 Cheng HM, Kim S, Park GH, Chang SE, Bang S, Won CH, et al. Low vitamin $\mathrm{D}$ levels are associated with atopic dermatitis, but not allergic rhi- nitis, asthma, or IgE sensitization, in the adult Korean population. J Allergy Clin Immunol. 2014 Apr;133(4):1048-55.

4 Amestejani M, Salehi BS, Vasigh M, Sobhkhiz A, Karami M, Alinia H, et al. Vitamin D supplementation in the treatment of atopic dermatitis: a clinical trial study. J Drugs Dermatol. 2012 Mar;11(3):327-30.

5 Manousaki D, Paternoster L, Standl M, Moffatt MF, Farrall M, Bouzigon E, et al. Vitamin D levels and susceptibility to asthma, elevated immunoglobulin E levels, and atopic dermatitis: A Mendelian randomization study. PLoS Med. 2017 May;14(5):e1002294. 